Pacific Journal of Mathematics

STER 


\title{
LEFT CENTRALIZERS AND ISOMORPHISMS OF GROUP ALGEBRAS
}

\author{
J. G. WENDEL
}

1. Introduction. Let $G$ be a locally compact group with right invariant Haar measure $m$ and real or complex group algebra $L(G)^{1}$. A bounded linear transformation $A$ of $L(G)$ to itself which commutes with all of the operations of left multiplication is called a left centralizer. Examples of transformations $A$ which satisfy the defining equation $A(x y)=x A y$, all $x, \mathrm{y} \in L(G)$, include

(i) right multiplications: $A x=x z$ for some fixed $z \in L(G)$;

(ii) right translations: $A=R_{g_{0}}=$ the operation of translation on the right by some fixed $g_{0} \in G$; and

(iii) convolutions with measures: $A x=x * \mu$, where $\mu$ is a countably additive set function of bounded variation defined on the Borel sets of $G$, and $x$ * $\mu$ is defined by

$$
(x * \mu)(g)=\int x\left(g h^{-1}\right) \mu(d h), \quad g \in G .
$$

It is clear that (i) and (ii) are special cases of (iii); thus, given $z \in L(G)$ we may define the appropriate $\mu$ by $\mu(E)=\int_{E} z(g) m(d g)$, while for an assigned $g_{0} \in G$ the corresponding measure is defined by $\mu(E)=0$ or 1 according as $E$ does not or does contain $g_{0}^{-1}$.

The principal result (Theorem 1) of Part I states that, conversely, every left centralizer is a convolution with a regular measure. Important auxiliary theorems (3 and 4) furnish a characterization of the right translations (up to scalar factors of unit modulus), and show that in the strong operator topology any left centralizer may be approximated by a finite linear combination of right translations.

In Part II these results are applied to obtain a generalization of a theorem proved in [5]. We showed there that if $T$ is an isometric isomorphism of (real,

${ }^{1}$ We are using the notation and terminology of [5]. In particular, for elements $x$ and $y$ of $L(G)$ the symbol $x y$ denotes the usual convolution-product.

Received September 2, 1951.

Pacific J. Math. 2 (1952), 251-261 
complex) $L(G)$ onto the (real, complex) algebra $L\left(G^{\prime}\right)$ belonging to a second locally compact group $G^{\prime}$ with right invariant Haar measure $m^{\prime}$, then there exists a topological isomorphism $\tau$ of $G$ upon $G^{\prime}$ and a continuous character $\chi$ such that $T$ is given by

$$
x^{\prime}\left(g^{\prime}\right) \equiv(T x)(\tau g)=c \chi(g) x(g)
$$

for each $x \in L(G)$ and almost all $g \in G, c$ being a measure-adjusting constant. ${ }^{2}$ In the present paper we obtain the existence of $\tau$ and the validity of $(*)$ by supposing only that $T$ is an isomorphism which does not increase norm. (Of course, once $\left(^{*}\right)$ is established it follows that $T$ must be an isometry after all.) This generalization was suggested by, and extends, some results of Helson [2], which the author had the privilege of reading in manuscript. Helson obtained the theorem to be proved here for the special case of abelian $G, G^{\prime}$ and complex algebras; his methods, unfortunately, seem to be essentially "abelian" in nature, in that he makes strong use of duality theory and the Bochner representation theorem for positive definite functions.

\section{Part I. Left Centralizers}

2. The principal theorem. We shall establish the following result:

THEOREM 1. Let $A$ be a left centralizer acting on (real, complex) $L(G)$. There exists a unique regular (real, complex) measure $\mu$ of bounded variation such that $A$ is giver by $A x=x * \mu$; furthermore, $\|A\|=\operatorname{var} \mu .^{3}$

Proof. Let $\left\{V_{\alpha}\right\}$ be a basis for the neighborhoods of the identity element of $G$, with $V_{\alpha}$ compact; we write $\alpha \leq \beta$ in case $V_{\alpha} \supseteq V_{\beta}$, so that $\{\alpha\}$ is a directed set. Let $e_{\alpha}$ denote the characteristic function of $V_{\alpha}$, normalized through division by $m\left(V_{\alpha}\right)$ so that $\left\|e_{\alpha}\right\|=1$. It is well known that $\left\{e_{\alpha}\right\}$ constitutes an approximate identity, in the sense that $\lim _{\alpha} x e_{\alpha}=x$ for each $x \in L(G)$. Applying the transformation $A$, we obtain

$$
\tilde{x} \equiv A x=A \lim _{\alpha} x e_{\alpha}=\lim _{\alpha} A\left(x e_{\alpha}\right)=\lim _{\alpha} x A e_{\alpha}=\lim _{\alpha} x f_{\alpha},
$$

where $f_{a} \equiv A e_{a}$ and $\left\|f_{a}\right\| \leq\|A\|\left\|e_{a}\right\|=\|A\|$.

The elements $f_{a}$ may be thought of as linear functionals on the space $C_{0}(G)$ of continuous functions vanishing at $\infty$; as such their norms are equal to their $L$ norms, and thus are bounded. Since the unit sphere in $C_{0}(G) *$ is weak*compact

${ }^{2}$ The notations $G^{\prime}, m^{\prime}$ used here replace $\Gamma, \mu$ of [5].

${ }^{3}$ Suggestions supplied by the referee have made possible considerable simplification in the proof of this result, as well as in that of Theorem 4. 
the functionals $f_{a}$ have a weak*limit point which, by the Kakutani-Riesz theorem is uniquely representable as a regular measure $\mu$, of bounded variation; in fact, $\operatorname{var} \mu \leq\|A\|$.

Let $K$ denote the subspace of $C_{0}(G)$ consisting of functions having compact support. If $x$ and $y$ belong to $K$, then so does the function $z$ defined by

$$
z(g)=\int y(h) x\left(h g^{-1}\right) m(d h) .
$$

Let $\epsilon>0$ and $\alpha_{0}$ be arbitrary; since $\mu$ is a weak*limit point of the $f_{\alpha}$, there is an $\alpha_{1}>a_{0}$ such that

$$
\left|\int z(g) \mu(d g)-\int z(g) f_{a_{1}}(g) m(d g)\right|<\epsilon \text {. }
$$

Replacing $z(g)$ by its definition and rearranging the iterated integrals, we get

$$
\left|\int y(h) m(d h) \int x\left(h g^{-1}\right) \mu(d g)-\int y(h) m(d h) \int x\left(h g^{-1}\right) f_{\alpha_{1}}(g) m(d g)\right|<\epsilon .
$$

Since $x f_{\alpha} \rightarrow \tilde{x}$ in $L(G)$ and therefore as functionals, we have, on passing to the limit through a suitable cofinal subset $\left\{\alpha_{1}\right\}$ of $\{\alpha\}$,

$$
\left|\int y(h) m(d h) \int x\left(h g^{-1}\right) \mu(d g)-\int y(h) \tilde{x}(h) m(d h)\right| \leq \epsilon .
$$

Therefore

$$
\int y(h) m(d h) \int x\left(h g^{-1}\right) \mu(d g)=\int y(h) \tilde{x}(h) m(d h)
$$

this shows that $x * \mu$ and $\tilde{x}$ are equal as functionals on $K$, hence on $C_{0}(G)$, and so finally as elements of $L(G)$.

Thus we have shown that $A x=x * \mu$, at least for $x \in K$; a density argument shows that the equation actually holds for all $x \in L(G)$. Since $\|A\| \leq \operatorname{var} \mu$ it follows from the reverse inequality above that $\|A\|=\operatorname{var} \mu$. If $x * \mu_{1}=x * \mu_{2}$ for all $x \in K$, then it is easy to see that $\mu_{1}=\mu_{2}$, from which it follows that $\mu$ is unique, and the proof is completed.

A result equivalent to Theorem 1 is the following:

THEOREM 2. In the strong operator topology, the set of convolution operators on $L(G)$ is a closed subset of the ring of all bounded operators.

Proof that Theorem 1 implies Theorem 2. Let $\left\{\mu_{a}\right\}$ be a directed sequence of convolution operators which converges to an operator $A$ in the strong topology. Clearly $A$ is a left centralizer, and the result follows.

Proof that Theorem 2 implies Theorem 1. As in the proof of Theorem 1, we 
have $A x=\lim _{a} x f_{a}$; but right multiplication by $f_{a}$ may be regarded as a convolution operator $\mu_{\alpha}$, and $\mu_{a}$ tends strongly to $A$. Hence $A$ is a convolution operator.

3. Auxiliary theorems. The right translations are characterized as follows:

Theorem 3. Let $A$ be a bounded linear mapping of $L(G)$ to itself. A has the form $A=\lambda R_{g_{0}}$ for some scalar $\lambda$ of unit modulus and some $g_{0} \in G$ if and only if

3A) A is a left centralizer; and

3B) A preserves norm. ${ }^{4}$

Proof. Since the mappings $\lambda R_{g_{0}}$ clearly have the indicated properties we have only to prove the reverse implication.

Let $\mu$ be the measure determined by $A$ in accordance with Theorem 1 . Then the assumption $3 \mathrm{~B}$ ) means that

$$
\int m(d g)\left|\int x\left(g h^{-1}\right) \mu(d h)\right|=\|x\|, \quad x \in L(G) .
$$

Let $|\mu|(E)=$ the total variation of $\mu$ on $E ;|\mu|$ is a regular measure and

$$
\left|\int x\left(g h^{-1}\right) \mu(d h)\right| \leq \int\left|x\left(g h^{-1}\right)\right||\mu|(d h) .
$$

We assert that equality holds in (2) for almost all $g$. Supposing the contrary, let strict inequality hold on a set of positive measure, and integrate (2) over $g$ with respect to $m$. From (1) we obtain, by means of Fubini's theorem,

$$
\begin{aligned}
\|x\| & =\int m(d g)\left|\int x\left(g h^{-1}\right) \mu(d h)\right|<\int m(d g) \int\left|x\left(g h^{-1}\right)\right||\mu|(d h) \\
& =\int|\mu|(d h) \int\left|x\left(g h^{-1}\right)\right| m(d g)=\int|\mu|(d h) \int|x(g)| m(d g) \\
& =(\operatorname{var} \mu)\|x\|=\|A\|\|x\|=\|x\| .
\end{aligned}
$$

This contradiction proves the assertion.

Let $x \in K$. Then both members of (2) are continuous functions of $g$, so that e quality holds everywhere; set $g=i=$ identity of $G$ and replace the function $x(h)$ by $x\left(h^{-1}\right)$, which is again an element of $K$. We obtain

$$
\left|\int x(h) \mu(d h)\right|=\int|x(h)||\mu|(d h) \equiv J(x) .
$$

${ }^{4}$ A similar result has been given by Kawada [4], for real $L(G)$. In Kawada's theorem the condition $\|A x\|=\|x\|$ is replaced by $A x \geq 0$ if and only if $x \geq 0$, where of course the inequalities are meant in the almost everywhere sense. The orem 3 can be deduced from this, and conversely. 
For $J(x) \neq 0$ and $x$ a nonnegative function, there is a unique constant $\lambda_{x}$ of unit modulus such that

$$
\int x(h) \mu(d h)=\lambda_{x} \int x(h)|\mu|(d h) .
$$

If $y$ is a nonnegative function in $K$ for which $J(y) \neq 0$, then similarly we may write

$$
\int y(h) \mu(d h)=\lambda_{y} \int y(h)|\mu|(d h),
$$

and

$$
\int\{x(h)+y(h)\} \mu(d h)=\lambda_{x+y} \int\{x(h)+y(h)\}|\mu|(d h) .
$$

But the left member of the last equation is also equal to

$$
\lambda_{x} \int x(h)|\mu|(d h)+\lambda_{y} \int y(h)|\mu|(d h) .
$$

Therefore $\lambda_{x}=\lambda_{y}=\lambda_{x+y}$. In other words, there is a unique constant $\lambda$ of unit modulus such that for all nonnegative $x \in K$ for which $J(x) \neq 0$ we have

$$
\int_{x}(h) \mu(d h)=\lambda \int_{x}(h)|\mu|(d h) .
$$

Hence for all Borel sets $E$ we have $\mu(E)=\lambda|\mu|(E)$, and we may as well suppose that $\mu$ is nonnegative. Equation (3) then becomes

$$
\left|\int x(h) \mu(d h)\right|=\int|x(h)| \mu(d h), \quad x \in K .
$$

By the regularity of $\mu$, this equation actually holds for all real continuous $x$ having limits at $\propto$; that is, for all $x$ in the space $C(G)$, where $G$ is made compact, if necessary, by adjoining an ideal point.

We now appeal to a theorem due to Kakutani [3], stating that, if $f$ is a functional of norm 1 on $C(\Omega)$ such that $x \geq 0$ implies $f(x) \geq 0$ and such that $\min (x$, $y)=0$ implies $\min (f(x), f(y))=0$, then $f$ is a point functional: $f(x)=x\left(\omega_{0}\right)$ for some fixed $\omega_{0} \in \Omega$. We apply the theorem to the functional $f(x)=\int x(h) \mu(d h)$. Clearly $\|f\|=1$, since var $\mu=1$. The functional is certainly order-preserving. Finally, if $\min (x, y)=0$ then $x(g)+y(g)=|x(g)-y(g)|$, and therefore

$$
\int\{x(g)+y(g)\} \mu(d g)=\int|x(g)-y(g)| \mu(d g)=\mid \int\{x(g)-y(g)\} \mu(d g)
$$

by (4). Consequently $f(x)+f(y)=|f(x)-f(y)|$, so that $\min (f(x), f(y))=0$.

The functional $\int_{x}(g) \mu(d g)$ is thus seen to satisfy the hypotheses of Kakutani's theorem, and therefore is a point functional, for some point other than the point at infinity, since the functional does not vanish identically on $K$. Therefore $\mu$ is concentrated at one point, and the operator $A$ has the desired form. 
Our next result states that the right translations span the space of all left centralizers. Precisely, we prove:

THEOREM 4. Every left centralizer $A$ is a strong limit point of the set of finite linear combinations of right translations.

Proof. By the Hahn-Banach theorem, if suffices to show that, it $F$ is any strongly continuous linear functional on the operators on $L(G)$, which vanishes on the right translations, then $F(A)=0$. A strongly continuous linear functional $F$ on the operators $\{T\}$ on a Banach space $X$ is given by an expression of the form

$$
F(T)=\sum_{i=1}^{n} x_{i}^{*}\left(T x_{i}\right)
$$

where $x_{i} \in X, x_{i}^{*} \in X^{*}, i=1,2, \cdots, n$ (see, for example, the proof of Theorem 2 in [1]). When $X=L(G)$, we have

$$
F(T)=\sum_{i=1}^{n} \int v_{i}(g)\left(T x_{i}\right)(g) m(d g)
$$

where the $v_{i}$ are bounded measurable functions.

Suppose now that $F$ vanishes on right translations. This means that

$$
\sum_{i=1}^{n} \int v_{i}(g) x_{i}\left(g h^{-1}\right) m(d g)=0 \quad(h \in G)
$$

Computing $F(A)$, we have

$$
\begin{aligned}
F(A) & =\sum_{i=1}^{n} \int v_{i}(g)\left(A x_{i}\right)(g) m(d g)=\sum_{i=1}^{n} \int v_{i}(g) m(d g) \int x_{i}\left(g h^{-1}\right) \mu(d h) \\
& =\int \mu(d h) \sum_{i=1}^{n} \int v_{i}(g) x_{i}\left(g h^{-1}\right) m(d g)=0,
\end{aligned}
$$

as we wished to show.

\section{Part II. Isomorphism of Group Algebras}

4. The isomorphism theorem. In this and succeeding $\S \S, G^{\prime}$ is a second locally compact group having right invariant Haar measure $m^{\prime}$, group algebra $L\left(G^{\prime}\right)$, and right translation operators $R_{g^{\prime}}^{\prime}$. The chief result to be established 
is the following:

THEOREM 5. Let $T$ be an algebra isomorphism of $L(G)$ onto $L\left(G^{\prime}\right)$, both algebras real or complex, which does not increase norms. There exists a bicontinuous isomorphism $\tau$ of $G$ upon $G^{\prime}$ and a continuous character $\chi$ (real or complex with $L(G)$ ) on $G$ such that
5A) $T R_{g} T^{-1}=\chi\left(g^{-1}\right) R_{\tau g}^{\prime}$, $g \in G, \tau_{g} \in G^{\prime}$
5B) $(T x)(\tau g)=c \chi(g) x(g)$;

hence $T$ is actually an isometry. The number $c$ is the constant value of the ratio $m(E) / m^{\prime}(\tau E)$.

The proof of Theorem 5, which will be given in $\$ 6$ after some necessary lemmas have been developed, is based on the following idea, due in part to Kawada [4]. First of all, it is clear that $T$ induces a $1-1$ mapping of the left centralizers of $L(G)$ onto those of $L\left(G^{\prime}\right)$ by means of the formula

$$
A^{\prime}=T A T^{-1},
$$

the boundedness of $T^{-1}$ being guaranteed by that of $T$ together with the 1 l-ness. In particular, then, translations on $L(G)$ are carried into left centralizers. It turns out, moreover, that the image of an arbitrary translation $R_{g}$ is an isometric left centralizer; this is proved in Lemma 1 . Therefore, by Theorem 3, it follows that the operator $T R_{g} T^{-1}$ is a scalar multiple of a translation on $L\left(G^{\prime}\right)$; we write

$$
T R_{g} T^{-1}=\lambda_{g} R_{\tau g}^{\prime}
$$

where $\left|\lambda_{g}\right|=1$. We then show (Lemmas 2,3 ) that $\tau$ is $1-1$, onto, and bicontinuous, and that $\lambda_{g}$ (or rather, its inverse) is a continuous character, thereby establishing 5A). The formula 5B) follows quickly with the aid of Theorem 4.

5. The mapping of translations. We shall first prove:

Lemma 1. Let $R_{g}$ be a right translation on $L(G)$ and set $Z_{g}^{\prime}=T R_{g} T^{-1}$. Then for some $\lambda_{g}$ of modulus unity, and for some $g^{\prime} \equiv \tau_{g} \in G^{\prime}$, we have

$$
Z_{g}^{\prime}=T R_{g} T^{-1}=\lambda_{g} R_{g}^{\prime} \cdot \text {. }
$$

Proof. In view of Theorem 3, we have only to show that $Z_{g}^{\prime}$ is isometric, since it certainly commutes with all left multiplications. As used in the proof of Theorem 1 , let $\left\{e_{\alpha}\right\}$ be an approximate identity of $L(G)$, and put $T e_{\alpha}=e_{\alpha}^{\prime}$. Choose $x^{\prime} \in L^{\prime}\left(G^{\prime}\right)$; clearly $x^{\prime} e_{\alpha}^{\prime} \rightarrow x^{\prime}$, since $x e_{\alpha} \rightarrow x$ for all $x \in L(G)$. Then 


$$
Z_{g}^{\prime} x^{\prime}=\lim _{\alpha} x^{\prime} Z_{g}^{\prime} e_{a}^{\prime}=\lim _{\alpha} x^{\prime} T R_{g} T^{-1} T e_{a}=\lim _{a} x^{\prime} T R_{g} e_{a},
$$

and therefore

$$
\begin{array}{r}
\left\|Z_{g}^{\prime} x^{\prime}\right\| \leq \varlimsup_{\lim _{\alpha}}\left\|x^{\prime} T R_{g} e_{a}\right\| \leq\left\|x^{\prime}\right\| \varlimsup_{\lim _{\alpha}}\left\|T R_{g} e_{a}\right\| \leq\left\|x^{\prime}\right\| \varlimsup_{\lim _{a}}\left\|R_{g} e_{a}\right\| \\
=\left\|x^{\prime}\right\| \overline{\lim }_{a}\left\|e_{\alpha}\right\|=\left\|x^{\prime}\right\|,
\end{array}
$$

which shows that $Z_{g}^{\prime}$ is a contraction. But $Z_{g}^{\prime-1}=Z_{g-1}^{\prime}$ is also a contraction, by the same argument. Therefore $Z_{g}^{\prime}$ is an isometry, as we had to show.

Lemma 2. The mappings $g \longrightarrow \lambda g$ and $g \longrightarrow \tau g$ defined above are continuous homomorphisms of $G$ to, respectively, the multiplicative group of scalars of unit modulus and the group $G^{\prime}$; $\tau$ is 1 - 1 .

Proof. The fact that the mappings are homomorphisms follows from the equations

$$
\lambda_{g h} R_{\tau(g h)}^{\prime}=T R_{g h} T^{-1}=T R_{g} T^{-1} T R_{h} T^{-1}=\lambda_{g} R_{\tau g}^{\prime} \lambda_{h} R_{\tau h}^{\prime}=\lambda_{g} \lambda_{h} R_{\tau g \tau h}^{\prime} .
$$

The function $\tau$ is $1-1$; for if $\tau g=i^{\prime}$, the identity of $G^{\prime}$, then

$$
\lambda_{g} l^{\prime}=T R_{g} T^{-1}, \text { and } R_{g}=\lambda_{g} l, \text { so that } g=i, \lambda_{g}=1 .
$$

In order to prove that $\tau$ is continuous, we observe that it is the product of the mappings $M_{1}, M_{2}, M_{3}$ defined by:

$$
\begin{array}{lr}
M_{1}: g \rightarrow R_{g}, & g \in G ; \\
M_{2}: R_{g} \rightarrow T R_{g} T^{-1}=\lambda_{g} R_{\tau g}^{\prime}, & g \in G ; \\
M_{3}: \lambda R_{g^{\prime}} \rightarrow g^{\prime}, & \lambda \text { a scalar of unit modulus, } g^{\prime} \in G^{\prime} .
\end{array}
$$

It is well known that, in the strong operator topology, $R_{g}$ is a continuous function of $g$, so that $M_{1}$ is continuous. $M_{2}$ is continuous, since $T$ and its inverse are bounded. The mapping $M_{3}$ is a homomorphism of the group of operators $\left\{\lambda R_{g}^{\prime} \cdot\right\}$ onto $G^{\prime}$; hence in order to prove its continuity everywhere it is sufficient to consider merely neighborhoods of the identity $l^{\prime}$.

Let $V^{\prime}$ be an arbitrary neighborhood of $i^{\prime} \in G^{\prime}$; we shall construct a strong neighborhood of $I^{\prime}$ whose image under $M_{3}$ is contained in $V^{\prime}$. Let $W^{\prime}$ be a neighborhood of $i^{\prime}$ having finite measure $w$ and satisfying $W^{\prime} W^{\prime-1} \subseteq V^{\prime}$. Let $x^{\prime} \in L\left(G^{\prime}\right)$ be the characteristic function of $W^{\prime}$. We shall show that if $\left\|\lambda R_{g^{\prime}}^{\prime} x^{\prime}-x^{\prime}\right\|<2 w$ then $g^{\prime} \in V^{\prime}$. In fact, suppose that $g^{\prime} \notin V^{\prime}$; then $W^{\prime}$ and $\mathbb{W}^{\prime} g^{\prime}$ are disjoint, so that 


$$
\begin{aligned}
\left\|\lambda R_{g}^{\prime} x^{\prime}-x^{\prime}\right\| & =\int\left|\lambda x^{\prime}\left(h^{\prime} g^{\prime}\right)-x^{\prime}\left(h^{\prime}\right)\right| m^{\prime}\left(d h^{\prime}\right) \\
& =\int\left\{\left|\lambda x^{\prime}\left(h^{\prime} g^{\prime}\right)\right|+\left|x^{\prime}\left(h^{\prime}\right)\right|\right\} m^{\prime}\left(d h^{\prime}\right) \\
& =\left\|\lambda R_{g}^{\prime}, x^{\prime}\right\|+\left\|x^{\prime}\right\|=2\left\|x^{\prime}\right\|=2 w .
\end{aligned}
$$

Hence $M_{3}$ and a fortiori $\tau=M_{3} M_{2} M_{1}$ are continuous in $g$. Finally, $\lambda_{g}$ is continuous since $\lambda_{g} l$ is the product of the uniformly bounded and continuous functions $T R_{g} T^{-1}$ and $R_{\tau_{g}-1}^{\prime}$.

LEMMA 3. The mapping $\tau$ exhausts $G^{\prime}$ and is a homeomorphism of $G$ onto $G^{\prime}$

Proof. We first show that $\tau G$ is closed in $G^{\prime}$. Suppose that $\left\{\tau g_{\alpha}\right\}$ is a directed sequence of elements which converges to an element $h^{\prime}$ of $G^{\prime}$. Then the corresponding translations $R_{\tau g_{a}}^{\prime}$ tend to $R_{h}^{\prime}$. Mapping back to the algebra of operators on $L(G)$, we see that $T^{-1} R_{\tau g_{\alpha}}^{\prime} T$ tends to $T^{-1} R_{h}^{\prime}, T$. But $T^{-1} R_{\tau g}^{\prime} T=$ $\lambda_{g}^{-1} R_{g}$ by definition of $\tau$ and $\lambda$. Therefore the operators $\lambda_{g_{a}}^{-1} R_{g_{a}}$ converge in the strong topology to an operator which is clearly on isometric left centralizer, and which therefore has the form $\lambda R_{h}$ for some scalar $\lambda$ and $h \in G$. Returning to $L\left(G^{\prime}\right)$, we readily see that $\lambda T R_{h} T^{-1}=R_{h}^{\prime}$, so that $h^{\prime}=\tau h, \lambda=\lambda_{h}^{-1}$.

Next we note that the continuity of $\tau^{-1}$ on $\tau G$ follows from the equation

$$
T^{-1} R_{g^{\prime}}^{\prime} T=\lambda_{\tau^{-1} g^{\prime}}^{-1} R_{\tau^{-1} g^{\prime}},\left(g^{\prime}=\tau g\right)
$$

just as that of $\tau$ was obtained from its defining equation.

Finally we establish the fact that $\tau G=G^{\prime}$. Suppose if possible that $h^{\prime} \in G^{\prime}$ has no counterimage in $G ; T^{-1} R_{h}^{\prime}, T \equiv A$ is nevertheless a left centralizer on $L(G)$, and therefore, by Theorem 4, may be expressed as the strong limit of a directed sequence $\left\{A_{a}\right\}$ of finite linear combinations of translations. Then $T A T^{-1}=R_{h}^{\prime}$ is the strong limit of operators $A_{a}$ each of which is a finite linear combination of translations $R_{g^{\prime}}^{\prime}$, with $g^{\prime} \in \tau G$. Let $W^{\prime}$ be a neighborhood of $i^{\prime}$ so small that $m^{\prime}\left(\mathbb{W}^{\prime}\right)<\infty$ and $h^{\prime} \mathbb{W}^{\prime-1} \mathbb{W}^{\prime} \cap \tau G$ is empty; the existence of such a $W^{\prime \prime}$ is assured by the fact that $\tau G$ is closed. Let $x^{\prime}$ be the characteristic function of $W^{\prime}$, and set $x_{\alpha}^{\prime}=A_{\alpha} x^{\prime}$. Let $k^{\prime}$ be any element of $W^{\prime} h^{\prime-1}$. Then $k^{\prime} g^{\prime} \notin \mathbb{W}^{\prime}$, for each $g^{\prime} \in \tau G$. Hence $x^{\prime}\left(k^{\prime} h^{\prime}\right)=1$ implies that $x_{\alpha}^{\prime}\left(k^{\prime}\right)=0$. Therefore

$$
\begin{aligned}
\left\|x_{a}^{\prime}-R_{h^{\prime}}^{\prime} x^{\prime}\right\| \geq \int_{W^{\prime} h,-1}\left|x_{\alpha}^{\prime}\left(k^{\prime}\right)-x^{\prime}\left(k^{\prime} h^{\prime}\right)\right| m^{\prime}\left(d k^{\prime}\right) & \\
& =\int_{W^{\prime} h^{\prime-1}} 1 m^{\prime}\left(d k^{\prime}\right)=m^{\prime}\left(W^{\prime}\right) .
\end{aligned}
$$

But this contradicts the assertion that $A_{\alpha}^{\prime}$ tends strongly to $R_{h}^{\prime}$. Hence no such $h^{\prime}$ can exist; this completes the proof of the lemma. 
6. Proof of the isomorphism theorem. If we define $\chi(g)=\lambda_{g}^{-1}$, it is clear that the lemmas of the preceeding $\S$ establish the existence and properties of $\tau$ and $\chi$ and the validity of $5 \mathrm{~A})$. In order to prove $5 \mathrm{~B}$ ), let $U$ be the isomorphism of $L(G)$ onto $L\left(G^{\prime}\right)$ which $5 \mathrm{~B}$ ) defines; that is,

$$
(U x)(\tau g)=c \chi(g) x(g), x \in L(G) .
$$

Then

$$
\left(U^{-1} x^{\prime}\right)(g)=c^{-1} \chi\left(g^{-1}\right) x^{\prime}(\tau g) \text { for } x^{\prime} \in L\left(G^{\prime}\right) .
$$

Hence

$$
\begin{aligned}
& \left(U R_{h} U^{-1} x^{\prime}\right)(\tau g)=c \chi(g)\left(R_{h} U^{-1} x^{\prime}\right)(g)=c \chi(g)\left(U^{-1} x^{\prime}\right)(g h)
\end{aligned}
$$

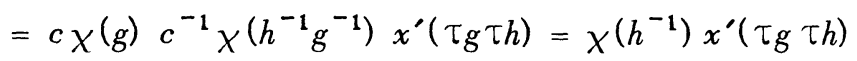

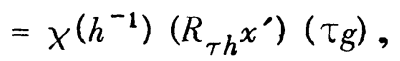

showing that $U R_{h} U^{-1}=\chi\left(h^{-1}\right) R_{\tau h}, h \in G$. Therefore $U R_{g} U^{-1}=T R_{g} T^{-1}$, and consequently $T^{-1} U R_{g}=R_{g} T^{-1} U$. Let $S=T^{-1} U$; we see that $S$ is a bicontinuous automorphism of $L(G)$ which commutes with all right translations. We shall show that $S$ is the identity mapping, which will prove that $U=T$, as desired.

Let $z \in L(G)$, and let $A$ be the left centralizer defined by right multiplication by $z: A x=x z$, all $x \in L(G)$. Let $\left\{A_{\alpha}\right\}$ be a directed sequence of combinations of translations which converges to $A$ in the strong topology. We have

$$
A x=\lim _{a} A_{\alpha} x .
$$

Therefore

$$
S A x=S \lim _{\alpha} A_{\alpha} x=\lim _{\alpha} S A_{\alpha} x=\lim _{\alpha} A_{\alpha} S x=A S x .
$$

In other words, using the fact that $S$ is an automorphism, we have

$$
S x S z=S(x z)=S A x=A S x=(S x) z,
$$

so that $S z=z$. Since $z \in L(G)$ is arbitrary, $S=I$, and the proof is completed.

\section{REFERENCES}

1. J. Dieudonné, La dualité dans les espaces vectoriels topologiques. Ann. Sci. Ecole Norm. Sup. (3) 58 (1942), 107-139.

2. H. Helson, Isomorphisms of abelian group algebras, to appear in Ark. Mat. 
3. S. Kakutani, Concrete representation of abstract (M) - spaces, Ann. of Math. (2) 42 (1941), 994-1024.

4. Y. Kawada, On the group ring of a topological group, Math. Japonicae 1 (1948), 1-5.

5. J. G. Wendel, On isometric isomorphism of group algebras, Pacific J. Math. 1 (1951), 305-312.

Yale University and RAND Corporation 



\section{PACIFIC JOURNAL OF MATHEMATICS}

\section{EDITORS}

R. M. RoBINSON

University of California Berkeley 4, California

\section{*R. P. Dilworth}

Califomia Institute of Technology Pasadena 4, California

\section{E. F. BECKENBACH, Managing Editor}

University of California

Los Angeles 24, California

*During the absence of Herbert Busemann in 1952.

\section{ASSOCIATE EDITORS}

\author{
R. P. DILWORTH \\ HERBERT FEDERER \\ MARSHALL HALL
}

\author{
P. R. HALMOS \\ HEINZ HOPF
}

R. D. JAMES

\author{
BØRGE JESSEN \\ PAUL LÉVY \\ GEORGE PÓLYA
}

\author{
J. J. STOKER \\ E. G. STRAUS
}

KÖSAKU YOSIDA

\section{SPONSORS}

UNIVERSITY OF BRITISH COLUMBIA

CALIFORNIA INSTITUTE OF TECHNOLOGY

UNIVERSITY OF CALIFORNIA, BERKELEY

UNIVERSITY OF CALIFORNIA, DAVIS

UNIVERSITY OF CALIFORNIA, LOS ANGELES

UNIVERSITY OF CALIFORNIA, SANTA BARBARA

OREGON STATE COLLEGE

UNIVERSITY OF OREGON

\author{
UNIVERSITY OF SOUTHERN CALIFORNIA \\ STANFORD UNIVERSITY \\ WASHINGTON STATE COLLEGE \\ UNIVERSITY OF WASHINGTON \\ AMERICAN MATHEMATICAL SOCIETY \\ NATIONAL BUREAU OF STANDARDS, \\ INSTITUTE FOR NUMERICAL ANALYSIS
}

Mathematical papers intended for publication in the Pacific Journal of Miathematics should be typewritten (double spaced), and the author should keep a complete copy. Manuscripts may be sent to any of the editors. All other communications to the editors should be addressed to the managing editor, E. F. Beckenbach, at the address given above.

Authors are entitled to receive 100 free reprints of their published papers and may obtain additional copies at cost.

The Pacific Journal of Mathematics is published quarterly, in March, June, September, and December, by the University of California, Berkeley 4, California. The price per volume (4 numbers) is $\$ 8.00$; single issues, $\$ 2.50$. Special price to individual faculty members of supporting institutions and to individual members of the American Mathematical Society: $\$ 4.00$ per volume; single issues, $\$ 1.25$.

Subscriptions, orders for back numbers, and changes of address should be sent to the publishers, University of California Press, Berkeley 4, California.

Printed at Ann Arbor, Michigan. Entered as second class matter at the Post Office, Berkeley, California.

\section{UNIVERSITY OF CALIFORNIA PRESS • BERKELEY AND LOS ANGELES}




\section{Pacific Journal of Mathematics}

\section{Vol. 2, No. $2 \quad$ February, 1952}

L. Carlitz, Some theorems on Bernoulli numbers of higher order...

Watson Bryan Fulks, On the boundary values of solutions of the heat equation........................................ 141

John W. Green, On the level surfaces of potentials of masses with fixed center of gravity................................... 147

Isidore Heller, Contributions to the theory of divergent series .......... 153

Melvin Henriksen, On the ideal structure of the ring of entire functions . . . 179

James Richard Jackson, Some theorems concerning absolute neighborhood retracts........................................ 185

Everett H. Larguier, Homology bases with applications to local connectedness ................................... 191

Janet McDonald, Davis's canonical pencils of lines ................ 209

J. D. Niblett, Some hypergeometric identities . . . . . . . . . . . . . . . . . . 219

Elmer Edwin Osborne, On matrices having the same characteristic equation...................................... 227

Robert Steinberg and Raymond Moos Redheffer, Analytic proof of the

Lindemann theorem ................................. 231

Edward Silverman, Set functions associated with Lebesgue area ......... 243

James G. Wendel, Left centralizers and isomorphisms of group algebras . . 251

Kosaku Yosida, On Brownian motion in a homogeneous Riemannian space. 